\title{
POLYLOGARITHMS AND POLY-BERNOULLI POLYNOMIALS
}

\author{
Abdelmejid BAYAD and Yoshinori HAMAHATA
}

(Received 18 February 2010)

\begin{abstract}
In this paper we investigate special generalized Bernoulli polynomials that generalize classical Bernoulli polynomials and numbers. We call them poly-Bernoulli polynomials. We prove a collection of extremely important and fundamental identities satisfied by our poly-Bernoulli polynomials and numbers. These properties are of arithmetical nature.
\end{abstract}

\section{Introduction and main results}

The classical polylogarithmic functions $\operatorname{Li}_{s}(x)$ are

$$
\operatorname{Li}_{s}(x)=\sum_{k=1}^{\infty} \frac{x^{k}}{k^{s}} .
$$

If $s$ is a negative integer, say $s=-r$, then the polylogarithmic function converges for $|x|<1$ and equals

$$
\mathrm{Li}_{-r}(x)=\frac{\sum_{j=0}^{r}\left\langle{ }_{j}^{r}\right\rangle x^{r-j}}{(1-x)^{r+1}},
$$

where the $\left\langle{ }_{j}^{r}\right\rangle$ are the Eulerian numbers. The Eulerian number $\left\langle{ }_{j}^{r}\right\rangle$ is the number of permutations of $\{1, \ldots, r\}$ with $j$ permutation ascents. One has

$$
\left\langle\begin{array}{l}
r \\
j
\end{array}\right\rangle=\sum_{\ell=0}^{j+1}(-1)^{\ell}\left(\begin{array}{c}
r+1 \\
\ell
\end{array}\right)(j-\ell+1)^{r} .
$$

We record $\mathrm{Li}_{-r}(x)$ for some $r$ :

$$
\begin{aligned}
\mathrm{Li}_{0}(x) & =\frac{x}{1-x}, & \mathrm{Li}_{-1}(x) & =\frac{x}{(1-x)^{2}}, \\
\operatorname{Li}_{-2}(x) & =\frac{x^{2}+x}{(1-x)^{3}}, & \mathrm{Li}_{-3}(x) & =\frac{x^{3}+4 x^{2}+x}{(1-x)^{4}}, \\
\operatorname{Li}_{-4}(x) & =\frac{x^{4}+11 x^{3}+11 x^{2}+x}{(1-x)^{5}}, & \mathrm{Li}_{-5}(x) & =\frac{x^{5}+26 x^{4}+66 x^{3}+26 x^{2}+x}{(1-x)^{6}} .
\end{aligned}
$$

2000 Mathematics Subject Classification: Primary 11B73, 11A07.

Keywords: polylogarithms; zeta functions; poly-Bernoulli numbers; poly-Bernoulli polynomials. 
From (1.2) we immediately deduce that, when $k$ is a negative integer, $\operatorname{Li}_{k}(x)$ is a rational function whose denominator is $(1-x)^{|k|+1}$. Precisely, for an integer $k \in \mathbb{Z}$, the formal power series $\operatorname{Li}_{k}(x)$ is the $k$ th polylogarithm if $k \geq 1$, and a rational function if $k \leq 0$. When $k=1$,

$$
\operatorname{Li}_{1}(x)=-\log (1-x) .
$$

Using $\operatorname{Li}_{k}(x)$, one can introduce (in the natural way) poly-Bernoulli numbers and polynomials.

For every integer $k$, we define a sequence of polynomials $\left(B_{n}^{(k)}(x)\right)_{n \in \mathbb{N}}$, which we call poly-Bernoulli polynomials, by

$$
\frac{\operatorname{Li}_{k}\left(1-e^{-t}\right)}{1-e^{-t}} e^{x t}=\sum_{n \geq 0} B_{n}^{(k)}(x) \frac{t^{n}}{n !} .
$$

We remark that $B_{n}^{(k)}(x)$ are defined in [3] by replacing $e^{x t}$ by $e^{-x t}$ in the left-hand side of (1.5). The numbers $B_{n}^{(k)}:=B_{n}^{(k)}(0)$ are the poly-Bernoulli numbers. These numbers are introduced by Kaneko [6], and then investigated in $[\mathbf{1}, \mathbf{2}, \mathbf{4}, \mathbf{5}, \mathbf{7 - 9}]$.

On the other hand, from (1.4) and (1.5), it is easy to see that, for any $n \geq 0$,

$$
(-1)^{n} B_{n}^{(1)}(-x)=B_{n}(x)
$$

where $B_{n}(x)$ are the classical Bernoulli polynomials given by their generating function

$$
\frac{t e^{x t}}{e^{t}-1}=\sum_{n=0}^{\infty} B_{n}(x) \frac{t^{n}}{n !}
$$

In this paper, we establish a series of properties, satisfied by our poly-Bernoulli polynomials and numbers, which are important and fundamental. These properties are of arithmetical nature.

THEOREM 1.1. (Explicit formula) For $k \in \mathbb{Z}, n \geq 0$, we have

$$
B_{n}^{(k)}(x)=\sum_{m=0}^{n} \frac{1}{(m+1)^{k}} \sum_{j=0}^{m}(-1)^{j}\left(\begin{array}{c}
m \\
j
\end{array}\right)(x-j)^{n} .
$$

THEOREM 1.2. (Recurrence formula 1) For all $k \geq 1, n \geq 0$, we have

$$
B_{n}^{(k)}(x)=\sum_{m=0}^{n}(-1)^{m}\left(\begin{array}{c}
n \\
m
\end{array}\right) B_{n-m}^{(k-1)} \sum_{l=0}^{m} \frac{(-1)^{l}}{n-l+1}\left(\begin{array}{c}
m \\
l
\end{array}\right) B_{l}(x) .
$$

THEOREM 1.3. (Recurrence formula 2) For $k \in \mathbb{Z}, n \geq 2$, we have

$$
B_{n}^{(k)}(x)=\frac{1}{n+1}\left\{B_{n}^{(k-1)}(x)+x B_{0}^{(k)}(x)-\sum_{m=1}^{n-1}\left(\left(\begin{array}{c}
n \\
m-1
\end{array}\right)-\left(\begin{array}{c}
n \\
m
\end{array}\right) x\right) B_{m}^{(k)}(x)\right\}
$$

and

$$
B_{0}^{(k)}(x)=1, \quad B_{1}^{(k)}(x)=\frac{1}{2}\left(B_{1}^{(k-1)}(x)+x B_{0}^{(k)}(x)\right) .
$$

THEOREM 1.4. (Appell sequence) For $k \in \mathbb{Z}, n \geq 0$, we have

$$
\frac{d}{d x} B_{n+1}^{(k)}(x)=(n+1) B_{n}^{(k)}(x) .
$$


THEOREM 1.5. (Addition formula) For $k \in \mathbb{Z}, n \geq 0$, we have

$$
B_{n}^{(k)}(x+y)=\sum_{m=0}^{n}\left(\begin{array}{l}
n \\
m
\end{array}\right) B_{m}^{(k)}(x) y^{n-m} .
$$

For $m, n \geq 0$, set

$$
C_{n}^{(-m)}(x, y)=\sum_{k=0}^{m}\left(\begin{array}{c}
m \\
k
\end{array}\right) B_{n}^{(-k)}(x) y^{m-k} .
$$

Then we have the following result.

THEOREM 1.6. (Symmetric formula) For $m, n$ and $C_{n}^{(-m)}(x, y)$ as above, we have

$$
\begin{aligned}
\sum_{n=0}^{\infty} \sum_{m=0}^{\infty} C_{n}^{(-m)}(x, y) \frac{t^{n}}{n !} \frac{u^{m}}{m !} & =\sum_{n=0}^{\infty} \sum_{m=0}^{\infty} C_{m}^{(-n)}(y, x) \frac{t^{n}}{n !} \frac{u^{m}}{m !} \\
& =\frac{e^{x t+y u} e^{t+u}}{e^{t}+e^{u}-e^{t+u}} .
\end{aligned}
$$

As a corollary, we have the following result.

THEOREM 1.7. (Duality) We have

$$
C_{n}^{(-m)}(x, y)=C_{m}^{(-n)}(y, x) .
$$

This is a dual relation for addition formula. In particular, for $x=y=0$, we obtain

$$
B_{n}^{(-m)}=B_{m}^{(-n)},
$$

the duality property of poly-Bernoulli numbers.

THEOREM 1.8. (Inversion formula) For $m, n \geq 0$, we have

$$
B_{n}^{(-m)}(x)=\sum_{k=0}^{m}\left(\begin{array}{l}
m \\
k
\end{array}\right) C_{n}^{(-k)}(x, y)(-y)^{m-k} .
$$

TheOREM 1.9. (Closed formula) For $m, n \geq 0$, we have

$$
C_{n}^{(-m)}(x, y)=\sum_{j=0}^{\infty}(j !)^{2}\left(\sum_{a=0}^{n}(x+1)^{n-a}\left(\begin{array}{l}
n \\
a
\end{array}\right)\left\{\begin{array}{l}
a \\
j
\end{array}\right\}\right)\left(\sum_{b=0}^{m}(y+1)^{m-b}\left(\begin{array}{l}
m \\
b
\end{array}\right)\left\{\begin{array}{l}
b \\
j
\end{array}\right\}\right),
$$

where

$$
\left\{\begin{array}{l}
n \\
m
\end{array}\right\}=\frac{(-1)^{m}}{m !} \sum_{l=0}^{m}(-1)^{l}\left(\begin{array}{c}
m \\
l
\end{array}\right) l^{n}
$$

are the so-called Stirling numbers of the second kind.

For $k \in \mathbb{Z}$ we set

$$
Z_{k}(s, x):=\frac{1}{\Gamma(s)} \int_{0}^{\infty} \frac{\operatorname{Li}_{k}\left(1-e^{-t}\right)}{1-e^{-t}} e^{-x t} t^{s-1} d t
$$

the Laplace-Mellin integral. It is defined for $\operatorname{Re}(s)>0$ and $x>0$ if $k \geq 1$, and for $\operatorname{Re}(s)>0$ and $x>|k|+1$ if $k \leq 0$. 
THEOREM 1.10. (Interpolation formula) The function $s \rightarrow Z_{k}(s, x)$ has analytic continuation to an entire function on the whole complex s-plane and

$$
Z_{k}(-n, x)=(-1)^{n} B_{n}^{(k)}(-x)
$$

holds for $n \geq 0$ and $x>0$. Besides, this zeta function can be rewritten as follows. For $k \in \mathbb{Z}$, we have

$$
Z_{k}(s, x)=\sum_{m \geq 0} \frac{1}{(m+1)^{k}} \sum_{j=0}^{m}(-1)^{j}\left(\begin{array}{c}
m \\
j
\end{array}\right) \frac{1}{(x+j)^{s}} .
$$

In the case $k \leq 0$, we have a more precise expression for this zeta function. For $k \leq 0$,

$$
Z_{k}(s, x)=\sum_{j=0}^{|k|}\left(\begin{array}{c}
|k| \\
j
\end{array}\right) \sum_{m=0}^{|k|-j} \frac{\left(\begin{array}{c}
|k|-j \\
m
\end{array}\right)(-1)^{m}}{(x+m-|k|-1)^{s}} .
$$

It should be noted that the first result of the above theorem has been independently obtained by Coppo and Candelpergher [3] and the present authors.

Remark 1.11. We can make the following two remarks.

(1) In particular, for $k=1$ we have

$$
\begin{aligned}
Z_{k}(s, x) & =\frac{1}{\Gamma(s)} \int_{0}^{\infty} \frac{\operatorname{Li}_{k}\left(1-e^{-t}\right)}{1-e^{-t}} e^{-x t} t^{s-1} d t \\
& =\frac{1}{\Gamma(s)} \int_{0}^{\infty} \frac{t}{1-e^{-t}} e^{-x t} t^{s-1} d t \\
& =\frac{\Gamma(s+1)}{\Gamma(s)} \frac{1}{\Gamma(s+1)} \int_{0}^{\infty} \frac{t^{s}}{1-e^{-t}} e^{-x t} d t \\
& =s \zeta(s+1, x),
\end{aligned}
$$

where $\zeta(s, x)$ is the Hurwitz zeta function. Then, for $k=1, s=-n$, we get from (1.6), (1.10) and (1.19) that

$$
Z_{1}(-n, x)=(-1)^{n} B_{n}^{(1)}(-x)=B_{n}(x)
$$

and

$$
Z_{1}(-n, x)=-n \zeta(1-n, x)
$$

Hence, we derive the famous classical interpolation relation:

$$
B_{n}(x)=-n \zeta(1-n, x)
$$

(2) For $k \leq 0$, from the formula (1.18) we derive another new explicit formula:

$$
B_{n}^{(k)}(x)=\sum_{j=0}^{|k|}\left(\begin{array}{c}
|k| \\
j
\end{array}\right) \sum_{m=0}^{|k|-j}\left(\begin{array}{c}
|k|-j \\
m
\end{array}\right)(-1)^{m}(x+m-|k|-1)^{n} .
$$

\section{Proofs of main results}

In this section we will give proofs of the theorems mentioned in the previous section. 
Proof of Theorem 1.1. Since

$$
\begin{aligned}
\frac{\operatorname{Li}_{k}\left(1-e^{-t}\right)}{1-e^{-t}} & =\sum_{m=1}^{\infty} \frac{\left(1-e^{-t}\right)^{m-1}}{m^{k}} \\
& =\sum_{m=0}^{\infty} \frac{\left(1-e^{-t}\right)^{m}}{(m+1)^{k}} \\
& =\sum_{m=0}^{\infty} \frac{1}{(m+1)^{k}} \sum_{j=0}^{m}(-1)^{j}\left(\begin{array}{c}
m \\
j
\end{array}\right) e^{-j t},
\end{aligned}
$$

we have

$$
\begin{aligned}
\frac{\operatorname{Li}_{k}\left(1-e^{-t}\right)}{1-e^{-t}} e^{x t} & =\sum_{m=0}^{\infty} \frac{1}{(m+1)^{k}} \sum_{j=0}^{m}(-1)^{j}\left(\begin{array}{c}
m \\
j
\end{array}\right) e^{(x-j) t} \\
& =\sum_{m=0}^{\infty} \frac{1}{(m+1)^{k}} \sum_{j=0}^{m}(-1)^{j}\left(\begin{array}{c}
m \\
j
\end{array}\right) \sum_{n=0}^{\infty}(x-j)^{n} \frac{t^{n}}{n !} \\
& =\sum_{n=0}^{\infty} \sum_{m=0}^{n} \frac{1}{(m+1)^{k}} \sum_{j=0}^{m}(-1)^{j}\left(\begin{array}{c}
m \\
j
\end{array}\right)(x-j)^{n} \frac{t^{n}}{n !} .
\end{aligned}
$$

This completes the proof.

Proof of Theorem 1.2. Since

$$
\frac{\operatorname{Li}_{k}\left(1-e^{-t}\right)}{1-e^{-t}}=\frac{e^{t}}{e^{t}-1} \int_{0}^{t} e^{-s} \frac{\operatorname{Li}_{k-1}\left(1-e^{-s}\right)}{1-e^{-s}} d s,
$$

we have

$$
\begin{aligned}
\sum_{n=0}^{\infty} B_{n}^{(k)}(x) \frac{t^{n}}{n !} & \left(\sum_{n=0}^{\infty} B_{n}(x) \frac{t^{n-1}}{n !}\right) \int_{0}^{t}\left(\sum_{n=0}^{\infty} \frac{(-s)^{n}}{n !}\right)\left(\sum_{n=0}^{\infty} B_{n}^{(k-1)} \frac{s^{n}}{n !}\right) d s \\
& =\left(\sum_{n=0}^{\infty} B_{n}(x) \frac{t^{n-1}}{n !}\right) \int_{0}^{t} \sum_{n=0}^{\infty} \sum_{m=0}^{n}(-1)^{n-m}\left(\begin{array}{l}
n \\
m
\end{array}\right) B_{m}^{(k-1)} \frac{s^{n}}{n !} d s \\
& =\left(\sum_{n=0}^{\infty} B_{n}(x) \frac{t^{n-1}}{n !}\right) \sum_{n=0}^{\infty} \sum_{m=0}^{n}(-1)^{n-m}\left(\begin{array}{c}
n \\
m
\end{array}\right) B_{m}^{(k-1)} \frac{t^{n+1}}{(n+1) !} \\
& =\sum_{n=0}^{\infty} \sum_{l=0}^{n} B_{n-l}(x) \sum_{m=0}^{l}(-1)^{l-m}\left(\begin{array}{c}
l \\
m
\end{array}\right) B_{m}^{(k-1)} \frac{t^{n}}{(l+1) !(n-l) !} \\
& =\sum_{n=0}^{\infty} \sum_{l=0}^{n} \frac{B_{n-l}(x)}{l+1}\left(\begin{array}{c}
n \\
l
\end{array}\right) \sum_{m=0}^{l}(-1)^{l-m}\left(\begin{array}{c}
l \\
m
\end{array}\right) B_{m}^{(k-1)} \frac{t^{n}}{n !} \\
& \text { by }\left(\begin{array}{l}
n \\
l
\end{array}\right)\left(\begin{array}{c}
l \\
m
\end{array}\right)=\left(\begin{array}{c}
n \\
m
\end{array}\right)\left(\begin{array}{c}
n-m \\
n-l
\end{array}\right)
\end{aligned}
$$




$$
\begin{aligned}
& =\sum_{n=0}^{\infty} \sum_{m=0}^{n} B_{m}^{(k-1)}\left(\begin{array}{c}
n \\
m
\end{array}\right) \sum_{l=m}^{n} \frac{(-)^{l-m}}{l+1}\left(\begin{array}{c}
n-m \\
n-l
\end{array}\right) B_{n-l}(x) \frac{t^{n}}{n !} \\
& \quad \text { put } l^{\prime}=n-l \\
& =\sum_{n=0}^{\infty} \sum_{m=0}^{n} B_{m}^{(k-1)}\left(\begin{array}{c}
n \\
m
\end{array}\right) \sum_{l^{\prime}=0}^{n-m} \frac{(-1)^{n-l^{\prime}-m}}{n-l^{\prime}+1}\left(\begin{array}{c}
n-m \\
l^{\prime}
\end{array}\right) B_{l^{\prime}}(x) \frac{t^{n}}{n !} \\
& \quad \operatorname{put} m^{\prime}=n-m \\
& =\sum_{n=0}^{\infty} \sum_{m^{\prime}=0}^{n} B_{n-m^{\prime}}^{(k-1)}\left(\begin{array}{c}
n \\
m^{\prime}
\end{array}\right) \sum_{l^{\prime}=0}^{m^{\prime}} \frac{(-1)^{m^{\prime}+l^{\prime}}}{n-l^{\prime}+1}\left(\begin{array}{c}
m^{\prime} \\
l^{\prime}
\end{array}\right) B_{l^{\prime}}(x) \frac{t^{n}}{n !} .
\end{aligned}
$$

The theorem therefore follows.

Proof of Theorem 1.3. Put $z=1-e^{-t}$. Then $t=\operatorname{Li}_{1}(z)$. From (1.5), we have

$$
\operatorname{Li}_{k}(z) e^{x \operatorname{Li}_{1}(z)}=z \sum_{n=0}^{\infty} B_{n}^{(k)}(x) \frac{\operatorname{Li}_{1}(z)^{n}}{n !} .
$$

Note that

$$
\frac{d}{d z} \operatorname{Li}_{k}(z)= \begin{cases}(1 / z) \operatorname{Li}_{k-1}(z) & (k>1) \\ 1 /(1-z) & (k=1) .\end{cases}
$$

Differentiating (2.1) with respect to $z$, we have

$$
\begin{aligned}
& \frac{1}{z} \operatorname{Li}_{k-1}(z) e^{x \operatorname{Li}_{1}(z)}+\operatorname{Li}_{k}(z) \frac{x}{1-z} e^{x \operatorname{Li}_{1}(z)} \\
& \quad=\sum_{n=0}^{\infty} B_{n}^{(k)}(x) \frac{\operatorname{Li}_{1}(z)^{n}}{n !}+\frac{z}{1-z} \sum_{n=0}^{\infty} B_{n+1}^{(k)}(x) \frac{\operatorname{Li}_{1}(z)^{n}}{n !} .
\end{aligned}
$$

Using $z /(1-z)=e^{t}-1$, we obtain

$$
\sum_{n=0}^{\infty} B_{n}^{(k-1)}(x) \frac{t^{n}}{n !}+x\left(e^{t}-1\right) \sum_{n=0}^{\infty} B_{n}^{(k)}(x) \frac{t^{n}}{n !}=\sum_{n=0}^{\infty} B_{n}^{(k)}(x) \frac{t^{n}}{n !}+\left(e^{t}-1\right) \sum_{n=0}^{\infty} B_{n+1}^{(k)}(x) \frac{t^{n}}{n !} .
$$

This yields

$$
\begin{gathered}
\sum_{n=0}^{\infty} B_{n}^{(k-1)}(x) \frac{t^{n}}{n !}-\sum_{n=0}^{\infty} B_{n}^{(k)}(x) \frac{t^{n}}{n !}+\sum_{n=0}^{\infty} \sum_{m=0}^{n}\left(\begin{array}{c}
n \\
m
\end{array}\right) x B_{m}^{(k)}(x) \frac{t^{n}}{n !} \\
=\sum_{n=0}^{\infty} B_{n}^{(k)}(x) \frac{t^{n}}{n !}-\sum_{n=0}^{\infty} B_{n+1}^{(k)}(x) \frac{t^{n}}{n !}+\sum_{n=0}^{\infty} \sum_{m=0}^{n}\left(\begin{array}{c}
n \\
m
\end{array}\right) B_{m+1}^{(k)}(x) .
\end{gathered}
$$

Comparing both sides of this identity gives

$$
B_{n}^{(k-1)}(x)-x B_{n}^{(k)}(x)+\sum_{m=0}^{n}\left(\begin{array}{l}
n \\
m
\end{array}\right) x B_{m}^{(k)}(x)=B_{n}^{(k)}(x)-B_{n+1}^{(k)}+\sum_{m=0}^{n}\left(\begin{array}{l}
n \\
m
\end{array}\right) B_{m+1}^{(k)}(x) .
$$

From this, we obtain

$$
(n+1) B_{n}^{(k)}(x)=B_{n}^{(k-1)}(x)+\sum_{m=0}^{n-1} x\left(\begin{array}{l}
n \\
m
\end{array}\right) B_{m}^{(k)}(x)-\sum_{m=1}^{n-1}\left(\begin{array}{c}
n \\
m-1
\end{array}\right) B_{m}^{(k)}(x),
$$

which leads to the result. 
Proof of Theorem 1.4. Differentiation of both sides of (1.5) with respect to $x$ gives

$$
\frac{t \operatorname{Li}_{k}\left(1-e^{-t}\right)}{1-e^{-t}} e^{x t}=\sum_{n=1}^{\infty} \frac{d}{d x} B_{n}^{(k)}(x) \frac{t^{n}}{n !} .
$$

From this, we have

$$
\frac{\operatorname{Li}_{k}\left(1-e^{-t}\right)}{1-e^{-t}} e^{x t}=\sum_{n=0}^{\infty}(n+1) \frac{d}{d x} B_{n}^{(k)}(x) \frac{t^{n}}{n !} .
$$

This completes the proof.

Proof of Theorem 1.5. From the definition in (1.5), we have

$$
\begin{aligned}
\sum_{n=0}^{\infty} B_{n}^{(k)}(x+y) \frac{t^{n}}{n !} & =\frac{\operatorname{Li}_{k}\left(1-e^{-t}\right)}{1-e^{-t}} e^{(x+y) t} \\
& =\frac{\operatorname{Li}_{k}\left(1-e^{-t}\right)}{1-e^{-t}} e^{x t} e^{y t} \\
& =\left(\sum_{m=0}^{\infty} B_{m}^{(k)}(x) \frac{t^{m}}{m !}\right)\left(\sum_{l=0}^{\infty} \frac{y^{l} t^{l}}{l !}\right) \\
& =\sum_{n=0}^{\infty}\left(\sum_{m=0}^{n}\left(\begin{array}{l}
n \\
m
\end{array}\right) B_{m}^{(k)}(x) y^{n-m}\right) \frac{t^{n}}{n !}
\end{aligned}
$$

The proof follows from this.

Proof of Theorem 1.6. Using the definition of $C_{n}^{(-m)}(x, y)$, the left-hand side can be rewritten as

$$
\begin{aligned}
\text { LHS } & =\sum_{n=0}^{\infty} \sum_{m=0}^{\infty} \sum_{k=0}^{m} B_{n}^{(-k)}(x) y^{m-k} \frac{t^{n}}{n !} \frac{u^{m}}{k !(m-k) !} \\
& =\sum_{n=0}^{\infty} \sum_{k=0}^{\infty} \sum_{l=0}^{\infty} B_{n}^{(-k)}(x) y^{l} \frac{t^{n}}{n !} \frac{u^{k}}{k !} \frac{u^{l}}{l !} \\
& =e^{y u} \sum_{n=0}^{\infty} \sum_{k=0}^{\infty} B_{n}^{(-k)}(x) \frac{t^{n}}{n !} \frac{u^{k}}{k !} \\
& =e^{y u} \sum_{k=0}^{\infty}\left(e^{x t} \sum_{n=0}^{\infty} B_{n}^{(-k)} \frac{t^{n}}{n !}\right) \frac{u^{k}}{k !} \\
& =e^{x t+y u} \sum_{k=0}^{\infty} \sum_{n=0}^{\infty} B_{n}^{(-k)} \frac{t^{n}}{n !} \frac{u^{k}}{k !} .
\end{aligned}
$$

Here, using the expression

$$
\sum_{k=0}^{\infty} \sum_{n=0}^{\infty} B_{n}^{(-k)} \frac{t^{n}}{n !} \frac{u^{k}}{k !}=\frac{e^{t+u}}{e^{t}+e^{u}-e^{t+u}}
$$

in Kaneko [6], the proof is completed. 
Proof of Theorem 1.8. To prove the theorem, we calculate the generating function $B_{n}^{(-m)}$.

$$
\begin{aligned}
\sum_{n=0}^{\infty} & \sum_{m=0}^{\infty}\left(\sum_{k=0}^{m}\left(\begin{array}{c}
m \\
k
\end{array}\right) C_{n}^{(-k)}(x, y)(-y)^{m-k}\right) \frac{t^{n}}{n !} \frac{u^{m}}{m !} \\
& =\sum_{n=0}^{\infty} \sum_{j=0}^{\infty} \sum_{k=0}^{\infty} C_{n}^{(-k)}(x, y) \frac{(-y u)^{j}}{j !} \frac{t^{n}}{n !} \frac{u^{k}}{k !} \\
& =e^{-y u} \sum_{n=0}^{\infty} \sum_{k=0}^{\infty} C_{n}^{(-k)}(x, y) \frac{t^{n}}{n !} \frac{u^{k}}{k !} \\
& =\frac{e^{x t+t+u}}{e^{t}+e^{u}-e^{t+u}} \\
= & \sum_{n=0}^{\infty} \sum_{m=0}^{\infty} B_{n}^{(-m)}(x) \frac{t^{n}}{n !} \frac{u^{m}}{m !} .
\end{aligned}
$$

This completes the proof.

Proof of Theorem 1.9. Using Theorem 1.6, we have

$$
\begin{aligned}
\sum_{n=0}^{\infty} & \sum_{m=0}^{\infty} C_{n}^{(-m)}(x, y) \frac{t^{n}}{n !} \frac{u^{m}}{m !} \\
& =\frac{e^{x t+y u+t+u}}{e^{t}+e^{u}-e^{t+u}} \\
& =\frac{e^{(x+1) t+(y+1) u}}{1-\left(e^{t}-1\right)\left(e^{u}-1\right)} \\
& =e^{(x+1) t+(y+1) u} \sum_{j=0}^{\infty}\left(e^{t}-1\right)^{j}\left(e^{u}-1\right)^{j} \\
& =\sum_{j=0}^{\infty} e^{(x+1) t}\left(e^{t}-1\right)^{j} e^{(y+1) u}\left(e^{u}-1\right)^{j} \\
& =\sum_{j=0}^{\infty}\left(j ! \sum_{n=0}^{\infty} \frac{(x+1)^{n} t^{n}}{n !} \sum_{m=0}^{\infty}\left\{\begin{array}{c}
m \\
j
\end{array}\right\} \frac{t^{m}}{m !}\right)\left(\begin{array}{l}
j ! \\
j !
\end{array} \sum_{n=0}^{\infty} \frac{(y+1)^{n} u^{n}}{n !} \sum_{m=0}^{\infty}\left\{\begin{array}{l}
m \\
j
\end{array}\right\} \frac{u^{m}}{m !}\right) \\
= & \left.\sum_{j=0}^{\infty}\left(j ! \sum_{l=0}^{\infty} \sum_{m=0}^{l}(x+1)^{l-m}\left(\begin{array}{c}
l \\
m
\end{array}\right)\left\{\begin{array}{l}
m \\
j
\end{array}\right\} \frac{t^{l}}{l !}\right)\left(\begin{array}{l}
n \\
k !
\end{array}\right) \sum_{p=0}^{\infty} \sum_{r=0}^{p}(y+1)^{p-r}\left(\begin{array}{l}
p \\
r
\end{array}\right)\left\{\begin{array}{l}
r \\
j
\end{array}\right\} \frac{u^{n}}{p !}\right) \\
= & \sum_{l=0}^{\infty} \sum_{p=0}^{\infty} \frac{\left.e^{u}-1\right)^{k}}{l !} \frac{u^{p}}{p !} \sum_{j=0}^{\infty}(j !)^{2}\left(\sum_{m=0}^{l}(x+1)^{l-m}\left(\begin{array}{l}
l \\
m
\end{array}\right)\left\{\begin{array}{l}
m \\
j
\end{array}\right\}\right)\left(\sum_{r=0}^{p}(y+1)^{p-r}\left(\begin{array}{l}
p \\
r
\end{array}\right)\left\{\begin{array}{l}
r \\
j
\end{array}\right\}\right)
\end{aligned}
$$

which proves the theorem. 
Proof of Theorem 1.10. Since the proof of the first part of the theorem is the same as that of Theorem 2 of Coppo and Candelpergher [3], we omit it.

We now prove (1.17) of Theorem 1.10. We have

$$
Z_{k}(s, x)=\frac{1}{\Gamma(s)} \int_{0}^{\infty} \sum_{n=1}^{\infty} \frac{\left(1-e^{-t}\right)^{m-1}}{n^{k}} e^{-x t} t^{s-1} d t .
$$

For $k \geq 1$, it is easy to see that, for all $N \geq 1$ and $t>0$,

$$
\begin{aligned}
\sum_{n=N}^{\infty} \frac{\left(1-e^{-t}\right)^{n-1}}{n^{k}} & \leq \sum_{n=N}^{\infty} \frac{\left(1-e^{-t}\right)^{n-1}}{n} \\
& \leq \sum_{n=1}^{\infty} \frac{\left(1-e^{-t}\right)^{n-1}}{n} \\
& =\frac{t}{1-e^{-t}}
\end{aligned}
$$

which ensures the convergence of the integral absolutely. Then we can invert $\int$ and $\sum$. Hence

$$
\begin{aligned}
Z_{k}(s, x) & =\frac{1}{\Gamma(s)} \sum_{n=1}^{\infty} \frac{1}{n^{k}} \int_{0}^{\infty}\left(1-e^{-t}\right)^{n-1} e^{-x t} t^{s-1} d t \\
& =\frac{1}{\Gamma(s)} \sum_{n=0}^{\infty} \frac{1}{(n+1)^{k}} \int_{0}^{\infty} \sum_{j=0}^{n}\left(\begin{array}{c}
n \\
j
\end{array}\right)(-1)^{j} e^{-(x+j) t} t^{s} d t \\
& =\sum_{n=0}^{\infty} \frac{1}{(n+1)^{k}} \sum_{j=0}^{n}(-1)^{j}\left(\begin{array}{c}
n \\
j
\end{array}\right) \frac{1}{\Gamma(s)} \int_{0}^{\infty} e^{-(x+j) t} t^{s-1} d t
\end{aligned}
$$

We thus obtain (1.17).

We prove the case $k \leq 0$. Since

$$
\operatorname{Li}_{k}(x)=\frac{1}{(1-x)^{|k|+1}} \sum_{j=0}^{|k|}\left(\begin{array}{c}
|k| \\
j
\end{array}\right) x^{|k|-j},
$$

we have

$$
\begin{aligned}
Z_{k}(s, x) & =\frac{1}{\Gamma(s)} \sum_{j=0}^{|k|} \int_{0}^{\infty}\left\langle\begin{array}{c}
|k| \\
j
\end{array}\right\rangle\left(1-e^{-t}\right)^{|k|-j} e^{(|k|+1) t} e^{-x t} t^{s-1} d t \\
& =\frac{1}{\Gamma(s)} \sum_{j=0}^{|k|}\left(\begin{array}{c}
|k| \\
j
\end{array}\right) \sum_{m=0}^{|k|-j}(-1)^{m}\left(\begin{array}{c}
|k|-j \\
m
\end{array}\right) \int_{0}^{\infty} e^{-(x+m-|k|-1) t} t^{s-1} d t .
\end{aligned}
$$

This yields (1.18) for all $k \leq 0, \operatorname{Re}(x)>|k|+1$.

Acknowledgement. We would like to thank the referee for informing us of the existence of [3]. The second named author is supported by a Grant-in-Aid for Scientific Research (No. 20540026), from the Japan Society for the Promotion of Science. 


\title{
REFERENCES
}

[1] T. Arakawa and M. Kaneko. Multiple zeta values, poly-Bernoulli numbers, and related zeta functions. Nagoya Math. J. 153 (1999), 1-21.

[2] T. Arakawa and M. Kaneko. On poly-Bernoulli numbers. Comment. Math. Univ. St. Pauli 48 (1999), 159-167.

[3] M.-A. Coppo and B. Candelpergher. The Arakawa-Kaneko zeta function. Ramanujan J. 22 (2010), 153-162.

[4] Y. Hamahata and H. Masubuchi. Recurrence formulae for multi-poly-Bernoulli numbers. Integers 7 (2007), A46.

[5] Y. Hamahata and H. Masubuchi. Special multi-poly-Bernoulli numbers. J. Integer Sequences 10 (2007), 07.4.1.

[6] M. Kaneko. Poly-Bernoulli numbers. J. Théor. Nombres 9 (1997), 221-228.

[7] M. Kaneko. Multiple Zeta Values and Poly-Bernoulli Numbers. Tokyo Metropolitan University Seminar Report, 1997.

[8] M.-S. Kim and T. Kim. An explicit formula on the generalized Bernoulli number with order $n$. Indian J. Pure Appl. Math. 31 (2000), 1455-1461.

[9] R. Sánchez-Peregrino. Closed formula for poly-Bernoulli numbers. Fibonacci Quart. 40 (2002), 362-364.

\author{
Abdelmejid Bayad \\ Département de Mathématiques \\ Université d'Évry Val d'Essone \\ Boulevard F. Mitterrand \\ 91025 Évry Cedex \\ France \\ (E-mail: abayad@maths.univ-evry.fr) \\ Yoshinori Hamahata \\ Graduate School of Mathematical Sciences \\ The University of Tokyo \\ 3-8-1 Komaba, Meguro, Tokyo \\ 153-8914 Japan \\ (E-mail:hamahata@ms.u-tokyo.ac.jp)
}

